\title{
Le processus d'observation, son développement et ses effets dans la méthode des autoconfrontations croisées en clinique de l'activité
}

The observation process, its development and its effects in the self-confrontation method

\section{Antoine Bonnemain, Emilie Perrot et Katia Kostulski}

\section{OpenEdition \\ Journals}

Édition électronique

URL : http://journals.openedition.org/activites/1111

DOI : 10.4000/activites. 1111

ISSN : 1765-2723

Éditeur

ARPACT - Association Recherches et Pratiques sur les ACTivités

\section{Référence électronique}

Antoine Bonnemain, Emilie Perrot et Katia Kostulski, « Le processus d'observation, son

développement et ses effets dans la méthode des autoconfrontations croisées en clinique de l'activité », Activités [En ligne], 12-2 | 2015, mis en ligne le 15 octobre 2015, consulté le 10 décembre 2020. URL : http://journals.openedition.org/activites/1111 ; DOI : https://doi.org/10.4000/activites. 1111

\section{(c) $($ () $\ominus$}

Activités est mis à disposition selon les termes de la licence Creative Commons Attribution - Pas d'Utilisation Commerciale - Pas de Modification 4.0 International 


\title{
Le processus d'observation, son développement et ses effets dans la méthode des autoconfrontations croisées en clinique de l'activité
}

\author{
Antoine Bonnemain \\ Équipe Psychologie du travail et clinique de l'activité, Centre de Recherche sur le Travail et le Développement \\ (CRTD, EA 4132), \\ Conservatoire National des Arts et Métiers \\ antoinebonnemain@yahoo.fr \\ Émilie Perrot \\ Équipe Psychologie du travail et clinique de l'activité, Centre de Recherche sur le Travail et le Développement \\ (CRTD, EA 4132), \\ Conservatoire National des Arts et Métiers \\ emilieperrot@hotmail.fr
}

Katia Kostulski

Équipe Psychologie du travail et clinique de l'activité, Centre de Recherche sur le Travail et le Développement (CRTD, EA 4132),

Conservatoire National des Arts et Métiers

katia.kostulski@cnam.fr

\begin{abstract}
The observation process, its development and its effects in the self-confrontation method. The purpose of this article is to analyze the development of observation as an activity within the crossed self-confrontation method. The first part describes the theoretical connections between observation and intersubjectivity in the observation process, and the psychological process that is involved. The second part presents an intervention based on the crossed self-confrontation in activity clinic. In this example, we analyze the effects of the observation process as it is perfomed among workers. We then study the migration of intersubjectivity in the observation process and its effects on the way that professionals might think about their work. More particularly, we suggest that observation leads professionals to "segment" their work activity, and thus to construct new objects of thought about work activity, through activity observation and analysis.
\end{abstract}

KEYWORDS

observation methods, intersubjectivity, autoconfrontation, intervention, clinic of activity.

\section{Introduction}

Notre contribution s'inscrit dans une réflexion sur les méthodologies en analyse du travail, dans une perspective inscrite en clinique du travail et plus particulièrement en clinique de l'activité. Nous souhaitons analyser et tenter de caractériser notre travail d'intervention dans des dimensions souvent peu visibles et peu déductibles de l'exposé des méthodes d'intervention. En effet, cette perspective s'inscrit dans une démarche clinique, et 
c'est une part de ce travail clinique et de ses effets qui sera l'objet de notre article: le moment, multiple et répété dans les interventions, de l'observation du travail, ses modalités, l'activité qui l'organise et ses effets chez les professionnels concernés et sur la dynamique de l'intervention. Dans cet article nous tentons d'étendre une définition de l'observation, en montrant comment l'activité d'observation se développe tout au long des différentes phases de la méthode d'analyse et de l'intervention. Plus encore, on voudrait montrer comment l'observation ne constitue pas seulement - même si c'est aussi toujours le cas - une méthode de recherche pour recueillir les données, mais aussi une méthode d'intervention dont le but premier est la transformation des situations de travail et des manières d'agir chez les professionnels.

Nous nous appuierons sur une intervention en clinique de l'activité auprès des chefs d'équipe des éboueurs d'une grande collectivité. Nous souhaitons caractériser la place que prend l'observation du travail à l'intérieur du processus d'élaboration individuel et collectif des critères que ces professionnels se fixent pour «bien faire» leur travail au quotidien. Nous montrerons en quoi l'observation du travail permet aux professionnels de produire de nouvelles ressources psychologiques pour l'action et en quoi elle les outille pour penser, ou repenser, les situations de travail dans lesquels ils agissent. Il s'agira d'instruire ici la question de l'usage qui est fait par les professionnels de l'observation dans la méthode d'analyse en autoconfrontations simples et croisées, en tant qu'instrument psychologique. De notre point de vue, cet usage va bien au-delà des observations initiales réalisées par le chercheur en situation de travail.

Nous instruirons cette question en deux temps. D'abord, nous discuterons différentes conceptions de l'observation, depuis la perspective positiviste chez Darwin, jusqu'aux méthodes qui intègrent la relation intersubjective et de ses effets en tant qu'ils sont constitutifs de l'observation elle-même. Ensuite, nous documenterons la question des effets de l'observation au cours de l'intervention lorsque ce sont les professionnels eux-mêmes qui s'y prêtent, comme c'est le cas en clinique de l'activité. Plus précisément, nous chercherons à montrer que l'observation permet aux professionnels d'objectiver les critères implicites de l'action, pour éventuellement les questionner, les reprendre ou les développer.

\section{1.- L'observation et son développement : du terrain à l'autoconfrontation croisée}

L'observation est une question pratique, méthodologique et théorique incontournable dans le champ de l'analyse du travail ou plus largement dans les sciences de l'homme. La diversité des conceptions théoriques qui l'entourent mérite qu'on s'y arrête, sans prétendre à l'exhaustivité, afin de définir notre périmètre d'étude. Ici, nous nous centrerons sur la fonction de l'intersubjectivité inhérente à l'observation et ses effets sur le développement de l'observation chez les professionnels eux-mêmes.

\section{1.- L'observation dans une perspective positiviste}

La dimension intersubjective liée à l'observation n'a pas toujours constitué, dans les sciences humaines, un objet en soi, et encore moins un instrument d'action. Dans la perspective positiviste, les travaux de Darwin en constituent une belle illustration. Darwin, dès 1838, pour étudier la manière dont les expressions émotionnelles ont émergé graduellement au cours de l'évolution, a effectué un nombre important d'observations sur les malades mentaux, les enfants et les animaux, en vue de construire une théorie évolutionniste de l'émotion (Rimé, 2005). Il a pour ce faire étendu le champ de ces observations à l'aide de questionnaires envoyés auprès de «missionnaires ou protecteurs d'indigènes », dans les régions les plus reculées du globe. Il formule ainsi son projet : «si les mêmes expressions et gestes se retrouvent [...] dans [les ethnies] qui ont eu peu de contact avec les Européens. Chaque fois que les mêmes mouvements du visage ou du corps expriment les mêmes 
émotions [...], on peut inférer avec une grande probabilité que de telles expressions sont réelles - c'est-à-dire innées ou instinctives » (Darwin, 1984, p. 137).

Dans cette étude, Darwin n'interroge ni l'observation en tant que méthode, ni les présupposés idéologiques qu'il mobilise sur les «indigènes » : il rapporte par exemple ces observations réalisées par des missionnaires: «un garçon Bengali fut accusé devant M. Scott de quelque méfait. Le coupable n'osa pas donner libre cours à sa colère en paroles, mais celle-ci fut tout à fait visible dans son expression, tantôt par le froncement des sourcils, tantôt par "un véritable rictus de chien" ». Dans ce rictus, «le coin de la lèvre au-dessus de la canine [...] était levé du côté de l'accusateur et s'accompagnait d'un fort froncement des sourcils» (1984, p. 138). Plus loin, il ajoute que «un missionnaire australien, M. Bulmer, répond à mon enquête: "Je découvre que les indigènes manifestent leur hargne les dents serrées, la lèvre supérieure tirée d'un côté, une expression générale de colère sur leur visage ; mais ils regardent leur interlocuteur en face" » (1984, p. 139).

Deux commentaires s'imposent. Premièrement, la perspective positiviste de ce travail d'observation scientifique cherche à établir l'observation à partir des «faits objectifs », mais la nature des notes d'observations, les expressions employées pour qualifier les éléments observés montrent à quel point toute observation s'ancre dans une interprétation, ici fortement reliée au contexte historique. Un «rictus de chien », ou encore «les indigènes manifestent leur hargne les dents serrées », sont utilisées ici en tant qu'éléments objectifs observés. Or, alors même que la posture positiviste devrait pousser l'investigation scientifique à questionner ce qui constitue un fait et ce qui constitue une interprétation, les termes permettant à Darwin de décrire les faits ne sont pas interrogés. Ils constituent une interprétation, en ce sens que rien d'autre, hormis le rapport idéologique à «l'indigène », ne semble pouvoir justifier l'observation de ces «faits objectifs». Pourtant, la conception positiviste, fondée par A. Comte, «postule l'objectivité du savoir scientifique, à partir des observations d'où l'on tire des énoncés singuliers dont la généralisation donnera naissance à des énoncés universels » (Ciccone, 2013, p. 18).

Deuxièmement, ces observations déléguées par Darwin lui permettent de soutenir un ensemble de thèses, notamment la thèse de «l'universalité de l'expression », qui postule que les émotions seraient les mêmes dans toutes les cultures. L'observation telle que mobilisée par Darwin ne fait pas état de la place de l'observateur dans la situation observée, la dimension d'interprétation des conduites observées, et moins encore les états internes de l'observé dans la situation. L'effet de l'observateur sur la conduite des individus observés n'a vraisemblablement pas constitué un objet de travail en soi. La relation entre celui qui observe et celui qui est observé, et les problèmes théoriques qu'elle pose, n'est jamais discutée par C. Darwin : l'observation est transparente. On peut comprendre dans le contexte de cette étude, qui à bien des égards, « est une véritable leçon sur la variété des méthodes qu'on peut mettre en œuvre quand on veut vérifier une hypothèse » (Rimé, 2005), que les avancées en termes méthodologiques restaient largement à construire.

Ce n'est que plus tard que certains auteurs se sont intéressés à la manière dont l'observation influence l'action.

\section{2.- L'observation comme construction d'un point de vue chez l'observateur}

Selon Bunge (1967/1984), «l'exactitude d'une observation n'est jamais garantie» (p. 54). L'intersubjectivité constitue de ce fait, pour lui - à l'inverse de Darwin - un objet de réflexion incontournable afin de penser les méthodes d'observation.

Pour l'auteur, philosophe et physicien, «l'observation est la procédure empirique de base » (Ibid., p. 47). Il existe selon lui deux types d'observations. Le premier est «l'observation directe » qui consiste à observer un objet directement perceptible; et le second, «l'observation indirecte » qui prend pour objet les phénomènes non directement 
observables, mais sur lesquels on peut réaliser un certain nombre d'inférences. Par exemple, l'observation des sentiments d'autrui est caractérisée par l'auteur comme observation indirecte, «inférence hypothétique, utilisant à la fois des données d'observation et des hypothèses » (p.48). Dans les deux cas, l'observation est dite « sélective et interprétative » : Sélective parce qu'elle prend appui sur un corps de connaissances qui détermine l'observation ; interprétative parce qu'elle tend à atteindre un but. Ces deux caractéristiques sont mêlées dans l'action d'observation, si bien que "nous sélectionnerons seulement les percepts qui nous paraissent pertinents par rapport à notre objectif et nous les replacerons dans nos connaissances » (Bunge, Ibid., p. 49). Il s'établit alors, si on suit cet auteur, un cycle de l'observation scientifique, entre d'une part la construction de connaissances sur l'objet, et d'autre part son observation toujours plus spécifique, à partir des connaissances acquises.

Ainsi, Bunge propose de concevoir l'intersubjectivité dans ce processus comme une condition nécessaire pour tendre vers une certaine objectivité : "l'intersubjectivité ne coïncide pas toujours avec l'objectivité, mais elle en est la condition» (p. 53). Puisque «l'exactitude d'une observation n'est jamais garantie» (p. 54), du fait de ses propriétés de sélection et d'interprétation, l'intersubjectivité entre ceux qui ont observé le même phénomène - les spécialistes de l'observation d'un objet - permet de développer, si l'on suit l'auteur, l'objectivité des phénomènes observés, en confrontant et en discutant les critères de sélection et d'interprétation que chacun a retenus dans la réalisation de l'observation.

On retiendra de ce travail l'idée que i) l'observation menée par un sujet adopte toujours un point de vue et que ii) ce n'est que lorsqu'elle est confrontée à d'autres points de vue qu'elle a quelque chance de devenir un peu plus objective. Il faut alors parler d'une véritable "activité d'observation", car le point de vue de l'observateur sur l'objet observé constitue un point de départ, et une résultante de cette activité. De plus, la connaissance produite par l'observation d'un objet - ici un objet le plus souvent «inanimé », dans le champ disciplinaire de l'auteur - s'appuie sur l'intersubjectivité, afin d'être en mesure de généraliser les résultats de l'observation.

Ce faisant, l'émergence d'un point de vue ne peut être regardée uniquement comme le résultat d'une activité individuelle, mais plutôt comme le résultat d'une co-activité, dans laquelle les observateurs entre eux font de l'intersubjectivité un moyen de construire ensemble une forme d' « objectivité ». Mais rappelons-le, l'intersubjectivité ne porte pas sur les rapports qui s'établissent dans le cours de l'observation entre celui qui observe et celui qui est observé.

Du côté des sciences humaines et sociales, cette conception de l'intersubjectivité comme moyen de l'objectivité vise à répondre à une critique importante et souvent reprise à propos de la méthode d'observation : le manque d'objectivité des résultats obtenus par observation d'un phénomène. Cette critique traverse les champs disciplinaires. Chapoulie (2000) reprenant l'histoire de l'apparition des recherches de terrain en sociologie, fait état des débats internes à la sociologie au début des années cinquante à propos de la méthode d'observation: «une première critique concerne l'objectivité des résultats, et porte notamment sur l'influence possible de l'observateur sur les données qu'il recueille: des données qui dépendent des singularités personnelles d'un observateur, des circonstances dans lesquelles il a travaillé, etc., ne sont pas susceptibles d'être à coup sûr obtenues par un autre chercheur dans la même situation» (p. 13). Un peu plus loin, il poursuit : «en fin de compte, selon ces critiques, les données recueillies par un travail de terrain sont peu susceptibles d'apporter des preuves solides à l'appui de propositions, si l'on prend pour référence le cadre logique de la démarche expérimentale. » (Ibid., p. 13). C'est à partir de ces débats que l'élaboration d'un cadre écologique de recueil des données a pu être développé, y compris en dehors de la sociologie. 


\section{3.- L'observation est une intersubjectivité}

En matière d'observation dans le champ de l'analyse du travail, c'est l'intersubjectivité entre le chercheur/observateur et le sujet observé qui fait le plus souvent l'objet des discussions. Pour certains en effet, les méthodes d'observation doivent permettre de ne pas transformer l'activité des sujets. C'est le cas par exemple des méthodes d'observation dans lesquelles l'observateur n'est pas visible. Dans ce cas, « l'observateur est soit caché (derrière une vitre sans tain par exemple), soit travaille à l'aide d'enregistrements vidéos. » (Norimatsu, 2014, p. 15). Pour d'autres, à l'inverse, l'observation transforme de fait l'activité. Dans ce cas, les rapports intersubjectifs entre l'observateur et l'observé jouent un rôle fondamental. C'est particulièrement le cas dans une conception de «l'observation clinique relationnelle » (Ciccone, 2013), qui fait du rapport intersubjectif un moyen d'élaboration et de pensée pour le sujet lui-même.

Droz (1984), en psychologie, a travaillé sur la place de l'intersubjectivité dans l'observation : « les observations qui appartiennent à l'espace subjectif de l'observateur sont des certitudes ou, du moins, elles peuvent l'être. Mais dès qu'elles pénètrent l'espace intersubjectif $[\ldots]$ elles exigent explication, argumentation, preuve [...] c'est l'interlocuteur, réel ou imaginaire, qui exige pour se laisser convaincre»(Ibid., p.8). On retrouve ici l'analyse menée par Bunge (1967/1984) : le social et l'objectivité vont de pairs. Mais R. Droz adopte un angle d'analyse sensiblement différent. Alors que Bunge réfère l'intersubjectivité à une confrontation entre observateurs, Droz, en tant que psychologue, la réfère plutôt aux rapports entre l'observateur et le sujet observé. C'est en exigeant de l'autre explication, argumentation et preuve, sur la base des observations qu'il réalise, que son observation prend un caractère intersubjectif. Elle peut acquérir par là même un certain degré d'objectivité, du moins plus grand qu'il ne l'était auparavant. De ce point de vue, le processus d'observation aurait donc un effet sur le réel observé tel que : «l'observateur n'est pas passif, et que le réel est, pour le moins, ré-actif» (p.9). Selon l'auteur «la relation psychologique - entre l'observateur et l'observé est nécessairement problématique [...] surtout parce qu'un individu qui devient observé change, consciemment ou non, de statut à l'égard de lui-même et à l'égard de l'autre. Il n'est plus seulement sujet, il est tout autant objet. » (p. 18). L'intersubjectivité conceptualisée par Bunge n'est donc pas une question qui se pose uniquement à propos des résultats de l'observation, entre experts des techniques et méthodes d'observations, mais aussi à l'intérieur de l'activité d'observation elle-même, entre le sujet/objet de l'observation, qui se forge une interprétation de la situation d'observation («pourquoi l'observateur m'observe-t-il ?...») et l'observateur, qui tente toujours et parfois malgré lui d'influer sur cette interprétation.

On peut conclure alors de manière provisoire que c'est dans un mouvement intersubjectif, que l'observation peut gagner en objectivité. L'objectivité de l'observation est liée au mouvement interprétatif qui se développe dans le rapport intersubjectif entre l'observateur et l'observé en situation d'observation.

Mais encore faut-il développer l'analyse des rapports intersubjectifs et de leur fonction au cours de l'activité d'observation. Suzanne Pacaud, dans le champ de l'analyse du travail, s'est intéressée, à sa manière, aux rapports entre l'observateur et l'observé. Dans son article consacré à «l'analyse psychologique et psychophysiologique du travail » (1954), l'auteure présente les bénéfices de ce qu'elle appelle la «méthode active », elle revient sur l'idée que les accidents et incidents survenant au cours du travail seraient liés, non pas aux traits de caractères des sujets, mais «à des associations perturbatrices fortuites ». Ces mêmes associations se déroulant à l'intérieur du sujet, elles « occupent toutes les voies nerveuses, empêchent les sensations informatives de circuler [...] Elles faussent l'importance relative des sensations, se substituent à elles et déclenchent par la force de leur charge affective, un acte non contrôlé. » (Pacaud, 1954, p.588). C'est pourquoi Suzanne Pacaud préconisait alors au psychologue, pour comprendre la nature de ces associations, «pour les saisir sur le vif», 
de passer par une phase d'apprentissage du métier, une phase d'assimilation et d'automatisation des techniques professionnelles, indispensables, selon elle, à toute analyse psychologique du travail. Pendant cette période d'apprentissage nécessaire du métier par le psychologue, quatre techniques devraient alors être employées pour garantir le caractère scientifique de l'étude du travail. Parmi ces techniques, l'auteur invoque "l'auto-observation et l'introspection au cours de l'apprentissage du métier, confrontées avec les témoignages des exécutants et les avis de techniciens ». Cette manière d'observer le travail peut sans doute être rapportée aux méthodes d'observations «directes» ou «participantes» développées depuis longtemps par la sociologie (voir sur ce point Arborio, 2007; ou Chapoulie, 2000).

«L'auto-observation » décrite par Suzanne Pacaud, nous permet d'éclairer l'examen de la méthode d'observation et des rapports intersubjectifs observateur/observé, ici du point de vue de la psychotechnique. Dans une étude réalisée auprès des téléphonistes de la SNCF, Pacaud indique que «le travail normal de la téléphoniste consiste à vaincre une série de difficultés »(Ibid., p. 599). Pour réaliser la technique d'auto-observation décrite précédemment, elle effectue un stage de quatre semaines à l'intérieur de ce service de la SNCF, ce qui lui permet de comprendre les difficultés du métier de téléphoniste du point de vue psychologique. Elle en dénombre 14, que nous ne pourrons décrire ici, qui vont du «manque d'articulation verbale » chez l'interlocuteur, à la gestion des plaintes parfois « tout à fait imméritées » que les usagers adressent aux téléphonistes.

Deux éléments de première importance se signalent dans le travail de Pacaud. Premièrement la nécessité de l'auto-observation vient du fait que les mécanismes psychologiques à l'œuvre dans le travail sont inobservables. Analyser le processus même de ces mécanismes requiert donc du psychologue un investissement sensible. Ce type d'investissement requiert une forme d'observation participante, c'est-à-dire un mode particulier «de présence du chercheur au sein du milieu observé »(Lapassade, 2002, p. 375). Deuxièmement, l'apprentissage du métier se fait sur la base, non pas uniquement de son effectuation, mais de son effectuation et des «confrontations » avec les exécutants et les techniciens du métier. L'observation prend, dans cette situation, le statut d'instrument psychologique permettant au psychologue d'accéder à ce qui n'est pas directement observable dans la situation. Il s'agit donc d'une utilisation indirecte de la méthode d'observation qui vise à lui faire éprouver par elle-même, et avec les autres, les difficultés du travail. On note l'importance ici de la dimension collective du travail qui semble se dégager de ses descriptions dans l'apprentissage des difficultés du métier. Par exemple, elle rapporte la difficulté à entendre les noms correctement au début de son apprentissage : "au début de notre stage, nous entendions les noms énoncés par les demandeurs sous la forme d'une véritable cacophonie dépourvue de sens. Le collationnement était matériellement impossible. Dès que ces noms nous ont été indiqués par les autres téléphonistes et plus tard bien connus, nous les percevions avec une grande netteté.»(Pacaud, 1954, souligné par nous, p. 605). L'observation, pratiquée de cette façon, était donc loin d'une observation impartiale et transparente. Elle engageait le psychologue dans un rapport intersubjectif aux professionnels, instrument du développement de ses compétences et de son expérience propres pendant le temps déterminé du stage. Il y a sans doute là de quoi soutenir l'idée d'une place de l'observateur construite dans l'usage qui est fait de la méthode d'observation. Autrement dit, en s'intégrant dans le milieu de travail, la psychologue agissait sur le milieu. Le bouleversement induit par sa présence en situation de travail permettait à Pacaud de mieux comprendre, de mieux expérimenter et par la suite, de mieux étudier, l'activité psychologique du sujet en situation. Il y a donc ici un usage - non théorisé - de la relation aux travailleurs en situation d'observation et de la place de l'observateur comme moyen d'analyse psychologique du travail. Mais dans le développement de cette activité d'observation telle que Pacaud la rapporte, on voit bien que ses effets ne se situent pas uniquement du côté du développement des compétences de la psychologue. Ils se situent tout 
autant du côté de l'activité des professionnels observés qui, dans cet exemple, est modifiée par la présence du psychologue, lorsqu'ils précisent les noms dont le collationnement constitue une difficulté. Une activité inhabituelle est convoquée par le psychologue, elle ne fait pas seulement l'objet d'un recueil de données. Dès lors, le cadre d'observation peut transformer l'activité des sujets observés. Il faut alors mieux comprendre en quoi l'observation, prise dans un rapport intersubjectif en situation, peut avoir pour effet de transformer l'activité des sujets observés.

\section{4.- L'observation comme moyen pour transformer l'activité du sujet : méthodologie en clinique de l'activité}

Dans le travail clinique inhérent à nos interventions, nous concevons l'observation comme un moyen de transformer le rapport subjectif au travail, ce qui ne laisse pas l'activité indemne. Nous cherchons également, via la phase d'observation notamment, à renverser le statut des professionnels dans l'intervention: d'une intervention «sur eux », à une intervention «pour et avec eux ». Comment décrire et qualifier cet usage de l'observation? En quoi l'intersubjectivité entre le chercheur et les professionnels constitue-t-elle un moyen du développement de l'activité d'observation chez les sujets au travail ?

La question est immense et fait l'objet de débats et de réflexions dans le champ de l'analyse du travail (voir par exemple Clot, 2005 ; Perrot, Bonnemain, \& Kostulski, 2013 ; Simonet, Caroly, \& Clot, 2011). Nous ne prétendrons pas ici y répondre exhaustivement, mais nous chercherons à établir des éléments à partir de données cliniques. Si la relation intersubjective entre l'observateur et l'observé « pèse » dans le cours des situations d'observation, il faut dès lors se préoccuper de savoir comment, sans nier l'existence de cette relation, on peut chercher à en faire quelque chose pour déplacer l'observation entre les professionnels, afin qu'elle devienne un instrument pour le développement de leur propre activité, et non plus seulement celle du chercheur. La méthode en autoconfrontation (Clot, Faïta, Fernandez, \& Scheller, 2001) peut constituer sur ce plan un levier clinique important.

La méthodologie historico-développementale dans laquelle nous nous situons part de l'idée, avec Vygotski, que «l'homme est plein à chaque minute de possibilités non réalisées»(2003, p. 76). Ce que le sujet réalise dans son activité n'est que l'une des possibilités réalisable dans la situation. C'est le sens de la distinction que nous faisons entre l'activité réalisée et le réel de l'activité. L'activité réalisée ne recouvre pas toute l'activité, il faut y ajouter ce que le sujet n'a pas réalisé, ce qu'il aurait pu faire autrement, mais qu'il a choisi de ne pas faire, ou encore ce qu'il souhaiterait pouvoir faire, mais qu'il ne réalise pas effectivement, et qui continue de peser dans son activité. L'activité réalisée dans les objets du travail n'a «pas le monopole du réel de l'activité [...] l'activité empêchée comme l'activité rêvée ne peuvent être écartées du champ de l'activité réelle » (Clot, 2008, p. 67). Entendue de cette manière, l'activité réalisée n'est qu'une composante de l'activité. Elle ne la résume pas. Ce qui ne s'est pas fait, mais qui aurait pu se faire, fait également partie de l'activité.

Pour Vygotski, les possibilités écartées lors de la réalisation de l'action ne sont pas accessibles directement. C'est pourquoi cet auteur a beaucoup insisté sur l'importance des «méthodes indirectes»(Vygotski, 2003, 1927/1999) permettant d'organiser le « redoublement de l'expérience vécue », afin que les sujets puissent transformer l'expérience vécue d'un objet, en objet d'une nouvelle expérience vécue. Sur ce point en particulier, Vygotski a critiqué le «dogme de l'expérience immédiate»(Vygotski, 1999) de la psychologie objective et de la psychologie subjective. L'une et l'autre proposent d'accéder directement à l'expérience vécue, soit par la voie expérimentale, soit par la voie introspective. La théorie vygotskienne constitue de ce point de vue une proposition méthodologique originale pour étudier le développement de l'activité, une troisième voie, entre les méthodes objectives et les méthodes subjectives. L'originalité du travail de 
Vygotski consiste sans doute dans la proposition qu'il formule pour dépasser l'opposition apparente entre l'objectivisme et le subjectivisme. Il propose de remplacer la causalité objective ou subjective par une causalité historique en psychologie (Ibid.), qui prendrait comme objet l'histoire du développement de l'activité. De ce point de vue la méthode permettant d'accéder au vécu ne peut être qu'indirecte : elle doit permettre au sujet de transformer son activité réalisée en ressource pour de nouvelles réalisations. Ce faisant, c'est le réel de l'activité qui peut s'en trouver modifié ou réorganisé.

Du coup, la méthodologie d'intervention doit tenir compte de cette distinction en permettant aux professionnels de dire quelque chose de ce qu'ils ont fait, afin qu'ils soient aussi en mesure de dire quelque chose sur ce qu'ils auraient pu faire. En rendant visible l'action réalisée comme l'une des possibilités de l'action - parmi d'autres - nous cherchons à favoriser chez les professionnels le développement de nouvelles actions. Le contexte de l'autoconfrontation croisée est conçu pour permettre ce développement de l'expérience vécue. Dans ce contexte - dialogique - les professionnels peuvent éventuellement faire de leur expérience vécue le moyen de vivre d'autres expériences (Clot, 2003). C'est « une telle expérience redoublée, qui permet à l'homme de développer des formes d'adaptation active » (Vygotski, 2003, p. 72) et c'est ce processus qui soutient le développement du pouvoir d'agir.

Dans cette perspective historico-développementale, l'intervention constitue une tentative pour donner, avec nos interlocuteurs, « une histoire à l'objectivité » dans le milieu de travail. Sur ce point, l'analyse de Volochinov est précieuse : «la vérité n'est éternelle qu'en tant qu'évolution éternelle de la vérité $»(1977$, p. 218). En posant des cadres dialogiques qui permettent de mettre les professionnels au travail sur leur travail, des vérités sont dites, qui peuvent toujours être reprises dans le cours des dialogues, de sorte que c'est aussi la subjectivité des points de vue sur l'objet réel qui est mise à l'épreuve. Ainsi, « en cherchant à repousser les limites de l'objectivité, ce sont aussi celles de la subjectivité qui reculent $\gg($ Clot, 2008, p. 212).

La distinction que nous faisons entre méthodologie et méthode est centrale ici. La méthode doit permettre de réaliser la méthodologie que nous avons explicitée. Elle doit constituer un moyen pour permettre aux sujets de faire de leur expérience vécue le moyen de faire de nouvelles expériences, dans une perspective développementale. Notons que cet objectif de l'autoconfrontation nous semble différer, au moins sur ce point, d'une autoconfrontation envisagée comme moyen "d'accélérer la construction, l'explicitation et la diffusion du savoir technique fondé sur la pratique » (Mollo, \& Falzon, 2004, p. 533). Les objectifs et les moyens de la méthode en autoconfrontation croisée que nous allons reprendre ensuite sont « guidés » par l'axe méthodologique historico-développemental.

$\mathrm{Vu}$ sous cet angle, la méthode et ses différentes étapes doivent permettre de répondre à ces objectifs méthodologiques. L'autoconfrontation constitue l'une des méthodes disponibles pour y parvenir. Elle comporte trois phases principales: l'observation du travail des professionnels, l'autoconfrontation simple et l'autoconfrontation croisée. Dans chacune de ces phases, l'observation et l'intersubjectivité prennent une place importante. Plus précisément, l'intersubjectivité change de place dans l'activité d'observation, à travers chaque phase de la méthode. Il faut dès lors mieux comprendre la place de l'observation dans la méthode en autoconfrontation croisée. Nous pensons que l'observation ne se dissout pas dans la première phase de la méthode, elle existe aussi ensuite, lorsque ce sont les professionnels eux-mêmes qui sont en situation de s'observer en train d'agir lors des autoconfrontations simples et croisées. 


\section{5.- La méthode en autoconfrontation : développer l'interprétation de la situation en développant l'activité d'observation chez les professionnels eux-mêmes}

Au cours de l'intervention en milieu de travail, nous cherchons à construire les conditions pour que ces effets se produisent, grâce notamment à la mise en place de la méthode en autoconfrontation croisée. Les trois phases de cette méthode sont déjà largement explicitées ailleurs (voir par exemple Clot et al., 2001 ; ou Dubosq, \& Clot, 2010), on en rappellera ici les principes généraux liés à l'observation.

La méthode pose comme point de départ l'idée que la transformation de l'organisation passe avant tout par une transformation de l'activité des protagonistes de l'organisation, professionnels de terrain et prescripteurs. De ce point de vue, la connaissance ne peut avoir pour finalité de produire, pour ceux qui dirigent, les savoirs qui leur permettront de modifier l'organisation. Notre tentative consiste plutôt à provoquer la modification, chez nos interlocuteurs, de leur point de vue sur l'organisation, afin qu'ils puissent agir autrement sur l'organisation pour la transformer. La méthode en autoconfrontation est conçue en ce sens comme un «espace-temps différent»(Clot et al., 2001) dans lequel le cadre dialogique favorise l'élaboration de la pensée à partir de l'expérience professionnelle. Dans chacune des phases de la méthode, l'observation et son développement chez nos interlocuteurs constitue l'un des buts fondamentaux. En effet, chacune des phases de la méthode fait de l'activité d'observation un moyen clinique pour que les professionnels développent leur propre interprétation de la situation. Ce qui compte ici, c'est moins la première observation que la seconde qui prend la première pour objet. Autrement dit, la méthode organise le déplacement de l'observation: si c'est d'abord le chercheur qui observe le travail des professionnels, la méthode doit permettre de déplacer le point de vue, afin que ce soit les professionnels euxmêmes qui soient à l'initiative des observations qu'ils peuvent faire de leur travail au cours même de l'autoconfrontation croisée.

Dans la première phase, on cherche à dénaturaliser la situation en inversant le statut de l'observateur. «En intriguant l'observé sur ce que l'observateur cherche à voir de son activité, il cherche à développer l'observation de l'observé sur sa propre activité » (Clot, 2008; Clot, \& Fernandez, 2005). De ce point de vue, le rapport intersubjectif observateur/observé constitue un instrument de travail pour l'observateur. C'est grâce à lui que «l'attention que le sujet sent fixer sur lui semble, par une sorte de contagion très élémentaire, l'obliger à s'observer. S'il est en train d'agir, l'objet de son action et l'action elle-même sont brusquement supplantés par l'intuition purement subjective qu'il prend de son propre personnage » (Wallon, 1983, p. 287). Cet effet de superposition transforme dès lors l'action de l'observé : «son geste de métier est refait dans un nouveau mouvement de réalisation qui s'adresse aux intentions de ce nouveau destinataire qu'est l'observateur » (Simonet et al., 2011, p. 120). Alors même qu'il doit réaliser son travail, la présence et les intentions de l'observateur orientent et modifient intrinsèquement son activité ordinaire. Du coup, l'activité, en situation d'observation, change de contexte. Elle est même prise dans plusieurs contextes à la fois : «pas seulement ici et maintenant, mais aussi après et ailleurs »(Clot, 2008). Cette «interférence des contextes» (Simonet, 2011) est au fondement du développement d'un dialogue intérieur chez l'observé devenu observateur de sa propre activité, et constitue un moyen pour que le sujet développe lui-même, et avec les autres, sa propre activité d'observation. C'est grâce aux étonnements de l'observateur à propos de ce qui est observable dans l'activité que le sujet observé peut «se parler à luimême » en convoquant la voix de l'observateur externe (Clot, 2008).

Ces considérations rejoignent celles proposées par Ciccone (2013). Pour cet auteur, la situation clinique d'observation suppose « une adresse » à autrui, présent ou absent de la situation d'observation, dans laquelle l'observateur prend une place particulièrement importante. En situation d'observation, l'activité du professionnel observé est d'abord 
adressée au chercheur. Ici « la réponse du clinicien, de son interprétation, sera évaluée [...] dans sa capacité à faire advenir d'autres paroles, d'autres événements suggérant d'autres interprétations » (p.36). Autrement dit, l'action de l'observateur, dans une perspective clinique, consiste à produire de nouvelles interprétations de la situation afin que le sujet puisse, à partir de celles-ci, élaborer sa propre interprétation de la situation. Cette élaboration prend la forme d'un dialogue intérieur, lors de la première phase d'observation du travail par le chercheur, que le sujet s'adresse à lui-même.

C'est pour ne pas laisser ce dialogue intérieur se refermer que les phases suivantes de la méthode ont été mises en place. Les autoconfrontations, simples et croisées, visent à conserver la «motricité du dialogue » (Clot, \& Faïta, 2000) né lors de l'observation de terrain. Lors de l'observation de terrain par le chercheur, le dialogue opère du niveau social, intersubjectif - entre l'observateur et l'observé - vers le niveau psychologique et intrasubjectif chez l'observé. Ce qui questionne d'abord le chercheur se met à questionner le sujet : « observé dans son travail, il s'observe en travaillant» (Clot, 2005, p. 3). Puis, alors que le sujet réalisait en situation d'observation une auto-observation adressée à lui-même, dans la phase d'autoconfrontation simple, il devient un observateur extérieur de son activité, en présence d'un tiers. Il doit alors adresser ses commentaires au chercheur. L'activité d'observation, qui permettait initialement au sujet de revitaliser le dialogue, en son «for intérieur », devient, dans ce nouveau contexte, une activité d'observation adressée à autrui. Cette dernière reprend alors son statut interpsychologique et intersubjectif.

Enfin, l'autoconfrontation croisée, qui réunit deux professionnels et le chercheur, doit permettre au dialogue intérieur de se renforcer via les échanges entre professionnels. «Le dialogue intérieur est encore plus intérieur après cette expérience », si on suit Clot (2008, p. 229). «Ce qu'il y a de plus personnel dans le dialogue s'est trouvé amplifié par la conflictualité dialogique du social » (Ibid., p. 229).

Dès lors, l'activité d'observation qui prend place dans la parenthèse de l'autoconfrontation croisée fait également de l'intersubjectivité un moyen de développer l'analyse. Mais cette intersubjectivité-là s'est transformée, à ce stade de la méthode, car elle se réalise entre les professionnels, et non plus uniquement entre un observateur externe - le chercheur - et un sujet observé. Dans le cours de l'autoconfrontation croisée, les travailleurs changent de place, ils sont tour à tour observateurs - lorsqu'ils ont pour tâche de commenter l'activité d'un collègue à l'aide des images filmées - puis observés - lorsque c'est leur propre activité qui est commentée.

Ici, la méthode, loin de constituer uniquement un mode du recueil de données pour le chercheur, permet aux sujets de développer leur interprétation de la situation vécue, en passant d'un contexte à un autre (situation d'observation de terrain, autoconfrontation simple, puis autoconfrontation croisée). À travers ces différentes situations, un rapport s'établit entre ce que les professionnels disent de ce qu'ils font (ce qu'ils se disent à euxmêmes, ce qu'ils disent au chercheur, ce qu'ils disent à leurs collègues), d'un côté, et ce qu'ils font de ce qu'ils disent, de l'autre. Cette traversée des contextes d'observation génère des interférences dans le dialogue intérieur du sujet qui doit ré-adresser son dire en fonction de ses interlocuteurs successifs.

$\mathrm{Du}$ coup, la question de l'observation du travail est décisive. Elle doit permettre l'objectivation momentanée de l'action réalisée afin que les professionnels puissent transformer cette action. Bakhtine (1984) décrivait déjà comment « une observation vivante, compétente, impartiale, à partir d'un point de vue quelconque, garde toujours sa valeur et sa signification. La partialité et la limitation d'un point de vue (d'un observateur), voilà quelque chose qui peut toujours être rectifié, complété, transformé (inventorié) à l'aide de cette même observation à partir d'un point de vue différent» (p. 334). En figeant momentanément l'activité sur ses réalisations effectives, l'observation permet la reprise du réalisé avec les sujets engagés dans l'analyse de leur travail. De cette manière, on expose l'action réalisée à 
sa reprise par les professionnels eux-mêmes, pour qu'ils soient en mesure d'en faire quelque chose pour les réalisations à venir. C'est l'objectif même de la méthode.

Dans cette perspective, l'intersubjectivité migre au cours du déploiement de la méthode en autoconfrontation. D'abord entre le chercheur et le professionnel (en situation d'observation), puis entre les professionnels eux-mêmes qui deviennent observateurs de leurs activités (en autoconfrontation simple puis croisée). Ces migrations fonctionnelles (Vygotski, 1978) de l'intersubjectivité s'accompagnent d'effets sur la capacité des professionnels à extraire de leur vécu subjectif des éléments de leur activité, à délimiter sur le plan psychologique un objet de leur activité qui, de source de pensée, devient ressource à la fois pour poursuivre l'observation et pour le développement de l'activité pratique (Kostulski 2011; Kostulski \& Clot, 2007).

C'est ce problème de la « délimitation » du réel au moyen de l'observation que nous allons à présent renseigner.

\section{2.- Les effets du développement de l'observation entre professionnels}

À partir des éléments précédemment présentés, nous souhaitons montrer que l'observation du travail concret, lorsqu'elle se réalise en autoconfrontation entre les professionnels, leur permet de «segmenter» leur propre activité. Autrement dit, l'observation, en circulant dans l'intersubjectivité, en faisant varier les destinataires de l'analyse, contribue à objectiver le réel, au moins temporairement. Nous défendons l'idée que l'observation sert aux professionnels pour délimiter entre eux ce qui peut faire objet, c'est-à-dire ce qui peut être « découpé » et détaché de l'expérience vécue unitaire pour être repensé puis réintroduit dans l'activité de chacun.

Pour alimenter cette conception de l'observation comme instrument psychologique de délimitation du réel, nous nous appuyons d'abord sur le travail de Politzer (1928/1967), à propos de l'émergence des «faits psychologiques». Nous reviendrons sur ces éléments à partir d'un dialogue entre deux chefs d'équipe qui a révélé entre eux un problème de métier commun : la question de la prise en compte ou non de l'engagement des éboueurs lors de l'activité de répartition des tâches. C'est une question de première importance dans l'activité managériale des chefs d'équipe, dans la mesure où elle renvoie au problème de la justice et de l'impartialité prétendue du manager de proximité.

Afin de déterminer les effets de l'observation opérée par les deux chefs d'équipe au plan individuel et collectif, nous analyserons enfin la manière dont le « découpage » de l'activité qui s'est effectuée au cours de cette première observation est repris par d'autres chefs d'équipe au cours des observations ultérieures réalisées dans l'intervention.

\section{1.- L'observation provoque une segmentation psychologique de l'activité}

Chez Politzer (1928/1967), l'objet principal de la psychologie est le «drame», terme qu'il utilise dans son sens le plus scénique. Il tente, par une critique des fondements de la psychologie d'alors, de faire revenir la psychologie dans une conception «concrète » de l'expérience vécue. L'objet de la psychologie, selon l'auteur, ne devrait pas être, par exemple, la mémoire en tant que telle, mais plutôt le souvenir, en tant qu'il constitue une partie du drame. C'est également la raison pour laquelle la psychologie développée par Politzer est fondamentalement une psychologie «culturelle»: dans l'étude du souvenir, on est amené à prendre en compte l'histoire du sujet dans sa singularité, ce qui n'est pas forcément le cas de l'étude de la mémoire, dont la signification s'éloigne d'emblée de l'expérience vécue concrète. Son objectif était de faire du «vivant»la préoccupation première de la psychologie dans l'étude des fonctions psychophysiologiques. 
Le drame et le fait psychologique constituent les deux notions centrales introduites par Politzer, et contribuent au rapprochement entre l'étude psychologique du vivant et l'expérience concrète des sujets. Selon l'auteur, le chercheur en psychologie doit avoir pour objectif de comprendre la singularité de l'action de l'homme à travers son expérience individuelle, constituée d'actes. Politzer récuse ainsi l'étude des seuls états fonctionnels abstraits du sujet. Il propose au contraire de réintroduire le vivant à l'intérieur de l'analyse des fonctions, et il recommande pour cela l'étude de l'acte à travers le récit du sujet : «Le fait psychologique étant un segment de la vie d'un individu singulier, ce n'est pas la matière et la forme d'un acte psychologique qui sont intéressants, mais le sens de cet acte, et cela ne peut être éclairé que par les matériaux que fournit par un récit le sujet lui-même » (Politzer, 1928/1967, p. 81). Autrement dit, entre les fonctions psychophysiologiques en tant que telles (abstraites) et la vie réelle, il y a l'acte, comme trait d'union permettant de réintroduire le sujet vivant en psychologie : "l'acte de l'individu concret, c'est la vie, mais la vie singulière de l'individu singulier, bref, la vie, au sens dramatique du mot» (Ibid., p. 51). L'étude du drame ne peut donc se faire qu'à la condition expresse de conserver, au plan scientifique, le sens de l'événement pour le sujet lui-même : «Mon fils pleure parce qu'on va le coucher. Voilà l'événement. Mais il n’y a là pour la psychologie classique que sécrétion lacrymale consécutive à une représentation contrariant une tendance profonde. C'est tout ce qui est arrivé. On a donc quitté le plan du "drame humain" dont l'auteur est l'individu concret, et on l'a remplacé par un drame abstrait» (Ibid., p. 52). Ainsi, si l'on suit l'auteur, les fonctions psychophysiologiques ne peuvent être étudiées en dehors de toute référence au sens qui leur est attribué par le sujet lui-même. Dans l'exemple précédent, c'est le sens que l'enfant pourrait éventuellement attribuer à l'événement du coucher qui compte, et qui pourtant ne fait pas partie de l'interprétation proposée par cette analyse psychologique.

Dans ce cadre, le «fait psychologique », seconde notion que nous empruntons à Politzer, est défini comme un segment du drame, c'est-à-dire un segment de la vie du sujet. On pourrait dire une expérience subjectivement segmentée. Si le drame, c'est la vie, le fait psychologique en constitue une partie conscientisée qui fait sens pour le sujet.

Nous soutenons que, dans le développement de l'observation du travail concret par des professionnels au cours de la co-analyse de leur travail, un certain nombre de faits psychologiques se constituent. L'observation de l'activité d'un collègue en autoconfrontation croisée produit une activité d'analyse entre les professionnels. Cette analyse consiste pour le sujet, parfois à son corps défendant, à isoler dans son activité ou celle de son collègue ce qui est habituellement aggloméré dans l'expérience vécue. Cet effet peut être rapporté à ce que Vygotski a déjà décrit à propos de la prise de conscience : «Percevoir les choses autrement c'est en même temps acquérir d'autres possibilités d'action par rapport à elles. Comme devant un échiquier : je vois autrement, je joue autrement. En généralisant un processus propre de mon activité, j'acquiers la possibilité d'un autre rapport à lui. C'est en gros comme si ce processus était sélectionné dans l'activité générale de ma conscience » (1997, p. 317). En autoconfrontation, le sujet sélectionne certains objets dans l'activité générale de sa conscience à partir de ses observations, afin de les opposer dans le dialogue aux arguments de son collègue, réels ou supposés. Ce processus constitue l'un des effets majeurs du processus décrit précédemment de développement de l'observation et de l'intersubjectivité entre les professionnels. Il s'agit d'une segmentation de l'activité, dans l'analyse, qui fait de chacun de ses segments un objet potentiel de dialogue pour les professionnels, individuellement et collectivement. Ils peuvent ainsi reprendre cet objet à leur compte, ou au contraire s'en défaire, s'en extraire, se positionner par rapport à lui, etc. Lorsque cet objet nouveau est construit dans l'analyse individuelle et collective du travail, il se met à exister pour chacun des professionnels et en chacun d'eux. Autrement dit, lorsqu'il émerge dans et par le dialogue entre les professionnels, il peut devenir un instrument incontournable pour que chacun d'entre eux puisse repenser son activité. Il condense, en quelque sorte, les problèmes concrets qui se posent aux professionnels lorsqu'ils réalisent leur activité, et leur 
permet de les conceptualiser sous la forme d'un objet générique créé dans le dialogue provoqué par l'observation.

La psychologie concrète de Politzer qui tente de s'ancrer dans le réel en partant de la singularité et du récit «n'admet pas la substitution du drame impersonnel au drame personnel » (p. 53), comme on l'a vu précédemment. Ce qui compte ici c'est le rapport entre le drame, les segments de drame (les faits psychologiques) et la manière dont on peut les étudier. Dans cette psychologie «concrète », «le psychologue aura alors quelque chose du critique dramatique : un acte lui apparaitra toujours comme un segment du drame qui n'a d'existence que dans et par le drame. Sa méthode ne sera donc pas une méthode d'observation pure et simple, mais une méthode d'interprétation » (p. 53).

En clinique de l'activité, se pose alors la question de comprendre comment le drame se «segmente» via l'interprétation de leur activité par les sujets eux-mêmes, c'est-à-dire comment émergent les faits psychologiques. Autrement dit, en quoi l'autoconfrontation, en déplaçant l'activité d'observation entre les professionnels, est aussi pour chacun d'eux une méthode d'interprétation de leur propre activité. Ce faisant, c'est en observant et en interprétant individuellement et collectivement leur activité de travail que se forment les faits psychologiques chez les professionnels et entre eux. Nous voudrions montrer ici que l'observation a, de ce fait, une fonction psychologique qui donne aux sujets les moyens de prendre comme objet de pensée certains segments isolés de leur activité.

\section{2.- Exemple de l'analyse de l'activité des chefs d'équipe des éboueurs}

Afin de rentrer dans l'analyse de ce processus d'observation et de ses effets chez des professionnels-chefs d'équipe, nous présenterons dans cette partie trois extraits de dialogue. Le premier est un dialogue entre Bakary et Alexandre, deux chefs d'équipe qui sont collègues dans le même atelier de travail. Ils réalisent, dans le cadre de l'intervention en clinique de l'activité, l'observation de leur travail en autoconfrontation croisée. Dans ce cadre, et comme on l'a vu précédemment, on considère la méthode comme un moyen d'observation et de "développement de l'interprétation de la situation chez les sujets euxmêmes » (Clot, 2008, p. 222). Au cours de cette observation entre eux, les échanges se tournent rapidement sur le problème des manières possibles de prendre en compte l'engagement des éboueurs au cours de la répartition des tâches. Plus précisément, nous verrons que c'est à cet endroit que se constitue entre eux un nouvel objet d'analyse, que nous qualifions, avec Politzer, de fait psychologique.

Nous décrirons, dans un second temps, la discussion collective réalisée entre 12 chefs d'équipe, après qu'ils aient visionné cet extrait d'autoconfrontation croisée.

Le deuxième extrait que nous présenterons est issu d'un dialogue en autoconfrontation simple avec Nadine, qui montre comment la segmentation opérée en amont existe et fonctionne dans sa propre activité d'analyse.

Enfin, le troisième extrait, issue d'une autoconfrontation croisée entre Julien et Nadine qui s'est déroulée peu de temps après, se propose d'illustrer la manière dont le fait psychologique peut devenir une ressource pour le développement de l'interprétation de la situation par les professionnels eux-mêmes.

Avant de rentrer dans l'analyse, nous présenterons de manière succincte l'intervention et son origine dans cette collectivité auprès des chefs d'équipe.

\subsection{1.- Une intervention auprès des chefs d'équipe des éboueurs d'une collectivité}

La commande d'intervention a été formulée par le service de propreté d'une grande collectivité. Comparé à l'ensemble des entités de la ville, ce service fait le constat d'un taux d'absentéisme anormalement plus élevé chez les éboueurs. Afin d'accompagner un plan de prévention de l'absentéisme mis en place pour les éboueurs, l'hypothèse est faite que l'activité de l'encadrement direct des éboueurs contient sans doute des éléments qui 
pourraient éclairer ce taux d'absentéisme. Cette commande pose donc comme point d'entrée les rapports entre l'activité des chefs d'équipe et l'absentéisme des éboueurs. Il s'agit, pour les décideurs de ce service, d'identifier les facteurs managériaux de risques d'absentéisme chez les éboueurs.

Après une première phase d'observations répétées dans le milieu de travail, nous avons pu mettre en discussion cette commande d'intervention auprès d'un collectif élargi de professionnels. Les chefs d'équipe ont beaucoup discuté de la formulation de la commande. De leur point de vue, cette formulation laisse entendre que les chefs d'équipe seraient responsables d'une perte d'engagement dans le métier chez les éboueurs. Or, selon eux, le problème doit être élargi aux marges dont ils disposent pour prendre soin de l'engagement des éboueurs. Comme le dira un chef d'équipe, « le travail de l'encadrement [de proximité] consiste justement à faire revenir les éboueurs le lendemain». Il s'agit pour les chefs d'équipe de soutenir l'engagement dans le travail, pour que les éboueurs ne se soustraient pas au travail par la voie de l'absentéisme. Nous avons cherché à prendre au sérieux le point de vue du collectif en prenant pour objet de l'analyse les manières possibles pour maintenir, entretenir, ou plus simplement, prendre soin de l'engagement des éboueurs dans leur travail.

C'est sur la base de cette commande et de cette demande du collectif que nous avons proposées de mettre en place un dispositif d'intervention en clinique de l'activité supportée par la création de plusieurs espaces de discussions à l'intérieur de l'organisation :

— un comité de pilotage composé par les responsables du service de prévention des risques professionnels, le directeur du service technique de la propreté, et l'encadrement supérieur des chefs d'équipes ;

— un collectif de 13 chefs d'équipe volontaires pour s'engager dans la co-analyse de leur travail en auto-confrontation croisée.

Au fil de l'intervention, la co-analyse a été orientée par les échanges en autoconfrontation entre les chefs d'équipe sur leur travail, par la réalisation de réunions collectives qui visaient à mettre en discussion les observations réalisées en situation de travail et lors des autoconfrontations, et par des comités de pilotages réguliers avec la direction, puis en présence des professionnels.

Deux dimensions de l'activité des chefs d'équipe ont été soumises à l'analyse, car elles sont considérées comme des moments « critiques » de l'activité, sur la question de l'engagement, pour les chefs d'équipe : l'activité de «mise en route »du travail des éboueurs, et l'activité de «préparation du tableau de répartition des tâches ». On ne présentera dans cet article que l'analyse de la répartition des tâches, car les extraits présentés ensuite s'y rapportent directement. Cinq autoconfrontations croisées ont été réalisées sur cette activité avec le collectif de chefs d'équipe. Nous avons réalisé d'abord des observations du travail de chacun des professionnels dans leurs ateliers de travail respectifs. Deux semaines de travail complètes ont été observées auprès de chacun des chefs d'équipe. Pendant l'observation, nous notions les faits observés concernant de multiples dimensions de l'activité: organisations générales des journées de travail, suivi des tableaux de répartitions des tâches d'un jour à l'autre, etc., et nous mettions ces observations en discussion avec le professionnel concerné de manière systématique. Nous avons produit ensuite un film de l'activité de chaque chef d'équipe lors de la «mise en route », au moment où il construit le tableau de répartition des tâches. Cette séquence d'activité filmée faisait l'objet des analyses en autoconfrontation simple, puis en autoconfrontation croisée avec un collègue et le chercheur. Enfin, chaque série d'autoconfrontations croisées était suivie d'une restitution des analyses au collectif qui pouvait poursuivre les discussions autour des problèmes soulevés. Ces réunions constituaient également un moyen pour préparer avec le collectif le comité de pilotage, en faisant le choix des séquences d'activité de travail et d'activité d'analyse filmées qui seraient adressées au comité de pilotage suivant sous la forme d'un montage.

Le travail du chef d'équipe consiste à répartir chaque jour entre les éboueurs une diversité de 
tâches (collecte des ordures ménagères, collecte des objets encombrants, balayage des voies publiques, désherbage des trottoirs, nettoyage des marchés découverts, nettoyage des trottoirs à la lance, etc.). Pour réaliser ce travail de répartition, le chef d'équipe utilise un « tableau de répartition » comprenant le nom de chaque éboueur de l'atelier, son groupe de roulement d'appartenance (roulement journalier de $6 \mathrm{~h}$ ou de $9 \mathrm{~h}$ ) et les tâches sur lesquelles il est affecté en première ou en seconde partie de journée ( $1^{\text {ère }}$ et $2^{\text {ème }}$ " tranche horaire $\left.»\right)$. Au cours de la réalisation de la co-analyse de cette activité, la question de l'équité dans la répartition des tâches est partagée par le collectif de chefs d'équipe engagé dans le dispositif. Il semble ainsi admis par tous l'idée que la répartition doit permettre à chaque éboueur de s'y retrouver, c'est-à-dire de pouvoir réaliser le plus fréquemment possible les tâches qu'il préfère. Lors de nos observations, tous les chefs d'équipe ont mentionné ce critère comme un critère important pour réaliser la répartition des tâches à partir du tableau de répartition. S'instaure donc l'idée, au fil des observations réalisées par le chercheur, puis par les professionnels en auto-confrontation simple, croisée, ou au cours des réunions collectives régulières, que la répartition des tâches doit être « juste », et que l'équité en est l'instrument privilégié. Cette idée a été reprise plusieurs fois au cours des autoconfrontations, par exemple : «dans les ateliers c'est comme partout, si on n' est pas équitable ça peut créer une mauvaise ambiance » (A., autoconfrontation croisée avec G.), "après s' il l'a fait la semaine dernière et qu'il y en a d'autres qui l'ont fait entre temps, que ce n' est pas toujours lui qui fait les corvées, ça va » (E. à propos d'un éboueur qui énonce sur le film d'activité que les tâches ne «tournent» pas suffisamment entre les éboueurs; autoconfrontation croisée avec J.). L'ensemble de nos observations, appuyées par ce que peuvent en dire les chefs d'équipe et les éboueurs, montrent que l'équité dans la répartition des tâches est un critère générique partagé entre les différents professionnels du collectif. Autrement dit, l'activité de répartition des tâches est pensée dans le collectif dans les termes de l'équité.

Au cours d'une autoconfrontation croisée, entre deux des chefs d'équipe du collectif, faisant suite à ce constat, émerge dans la conversation un désaccord entre eux sur certains critères de la répartition des tâches. Ainsi, «être juste dans la répartition des tâches » recouvre en réalité une multitude de critères, qui entrent parfois en contradiction, et qui déterminent l'activité réalisée et les différences qu'ils constatent dans leurs manières de construire le tableau de répartition des tâches.

Cette première controverse sur les critères de répartition entre les deux professionnels, Bakary et Alexandre, a marqué le début, dans l'intervention, d'une analyse étendue du conflit entre chefs d'équipe sur les critères de qualité de la répartition des tâches.

C'est dans ce cadre que nous soutenons que l'observation permet au collectif professionnel de faire émerger des faits psychologiques collectivement débattus - en tant que segments de leur activité - pour être en mesure de se positionner par rapport à eux. Les échanges collectifs portant sur les faits psychologiques - ici les critères de la répartition des tâches peuvent ensuite, et éventuellement, être repris par chacun pour poursuivre son propre dialogue intérieur sur les questions vives du métier, et ce afin de repenser et de reprendre à nouveaux frais leurs manières de réaliser la répartition des tâches.

\subsection{2.- La segmentation de l'activité dans le dialogue à partir d'un problème de métier}

L'analyse de l'activité repose, on l'a vu, sur différents moments d'observations successifs en clinique de l'activité. D'abord observation par le chercheur en situation de travail, puis observation par le professionnel en situation d'extériorité (autoconfrontation simple), et enfin observation entre deux professionnels (autoconfrontation croisée). Nous cherchons à caractériser ce que ce cheminement dans la méthode peut produire au plan psychologique chez les professionnels concernés. Nous le ferons ici à partir d'un dialogue en autoconfrontation croisée entre Alexandre et Bakary, dans lequel se réalise une segmentation de leur activité. 
Situons l'extrait dans le cadre de cette séance de travail d'une durée d'environ $2 \mathrm{~h}$. Le film de l'activité de mise en route de Bakary a déjà été visionné en début d'autoconfrontation. $\mathrm{Au}$ bout d'une heure, le chercheur propose de passer au visionnage du film d'activité d'Alexandre.

L'activité filmée présente Alexandre lors de la mise en route du travail des éboueurs, le matin à partir de $6 \mathrm{~h}$. Il commence par réaliser un ensemble de tâches administratives pour être en mesure plus tard de sortir sur le terrain afin d'anticiper le travail à réaliser. Petit à petit, les éboueurs arrivent à l'atelier. La plupart d'entre eux rentrent dans le bureau du chef d'équipe pour le saluer. De manière très fréquente, c'est aussi l'occasion pour eux de visualiser le tableau de répartition des tâches pour voir sur quelles tâches ils ont été affectés ce jour-là. Ce moment est important pour Alexandre, car il lui permet d'identifier les présents pour constituer l'affectation. Pour cette raison, il n'affiche pas le tableau avant l'heure de départ au travail (à $6 \mathrm{~h} \mathrm{10}$ ) pour être en mesure de modifier sa répartition en cas d'absence imprévue.

Alors que le film se déroule, le chercheur arrête les images sur le tableau de répartition des tâches réalisé par Alexandre le jour de l'observation filmée. Il tente de questionner les conflits sous-jacents à la configuration de cette répartition des tâches entre éboueurs, sans savoir précisément en quoi consistent ces conflits : «j'aimerai bien qu'on discute un peu de ce tableau... là ». Alexandre précise alors ce qu'il serait possible de «discuter » à partir du tableau : «Alors... sur le, sur le groupe $7 \ldots$ si ! J'avais mis D. sur la $11 / 12 \ldots$ en $\mathrm{PE}^{1}$ ». Il poursuit : « $\cdots$ au lieu de la $\mathrm{VE}^{2}$ alors que c'était son premier... ou son deuxième neuf ${ }^{3}$ je crois...».

Dans la poursuite de cette analyse réalisée par Bakary et Alexandre, s'engage entre eux une controverse professionnelle sur la manière de répartir les tâches à propos d'un éboueur en particulier (D.). Les deux professionnels réalisent dans le dialogue une opposition entre deux critères possibles de la répartition des tâches, deux manières possibles de prendre en compte, plus ou moins, l'engagement des éboueurs lors de la répartition des tâches : «l'équité » ou «l'égalité ». À partir de l'extrait $n^{\circ} 1$, on se propose d'analyser comment se produit la segmentation de l'activité des chefs d'équipe à partir de la mise en discussion du conflit de critères «équité »/« égalité » au cours de la répartition des tâches. Nous chercherons à proposer une interprétation de ce qui se joue dans le processus de segmentation que nous avons décrit précédemment. Nous proposerons cette interprétation en tentant de mieux caractériser, du point de vue de l'activité d'observation à laquelle ils se livrent, ce qui a permis à Bakary et Alexandre d'opérer la distinction entre les deux termes. Nous verrons ensuite comment cette segmentation peut, au niveau collectif, devenir un instrument de l'activité d'analyse des autres chefs d'équipe engagés dans le dispositif d'intervention.

1 PE renvoie à la tâche de collecte des «Papiers-Emballages », c'est-à-dire des « bacs jaunes »

2 VE renvoie à la tâche de collecte sur «Voies Etroites », il existe plusieurs déclinaisons pour cette tâche, telles que VE-PE (Papiers-Emballages sur Voies Etroites) ou VE-OM (Ordures Ménagères sur Voies Etroites)

3 La semaine est organisée selon le roulement suivant : trois journées de 9h (6h-17h), suivies de deux journées de $6 \mathrm{~h}$ (6h-12h), suivies de deux journées de repos. 
1. Chercheur : Donc... toi tu trouves que c'est... que c'est plus efficace quoi de mettre cet agent-là [sur la VE] plutôt que... si il y a un problème il va pouvoir...

2. Bakary : Ben... le régler... et c'est vrai que, nous notre grand souci c'est que voilà les les collectes soient finies quoi. Après si on se retrouve plusieurs fois les collectes non terminées euh...

3. Alexandre : Oui, mais comme tu l'as dit ça n'est arrivé qu'une fois.

4. Bakary : Ouais

5. Alexandre : Ce n'est arrivé qu'une fois.

6. Bakary : Ouais... mais ça fait pas longtemps qu'on a... qu'on a opté ce changement.

7. Alexandre : C'est vrai. Mais sur la totalité ça...

8. Bakary : Nan, mais je te comprends dans ta... dans ta logique. Bon... on est responsables d'atelier c'est vrai qu'on devrait tous les traiter euh... de façon équitable...

9. Alexandre : Ou égale ouais.

10. Bakary : Voilà égale... donc euh...

11. Alexandre : Moi j'aime pas le mot équitable... j'suis désolé.

12. Chercheur : Non, mais... tu... égale ça te dirait ça ?

13. Alexandre : Egale je préfère que équitable

14. Chercheur : Et donc toi ton souci c'est que ce soit équitable quoi ?

15. Alexandre : Nan, que ce soit égal...

16. Chercheur : Oui... pardon...

(rires)

17. Alexandre : Ah non, mais...

18. Chercheur : Égal... donc ça veut dire que si...

19. Bakary : Pourquoi pas équitable? Pourquoi pas équitable?

20. Chercheur : Pourquoi pas équitable?

21. Alexandre : Equitable? Parce que la notion d'équité euh... par sa propre définition autorise que les traitements soient différents en fonction de qui fait quoi, et ça ça me convient pas... moi ce que je veux c'est que le traitement soit égal.

22. Chercheur (à Bakary) : Mais toi tu dirais équitable toi ?

23. Bakary : Ben oui...

24. Alexandre : Donc moi je dirai plutôt égal, moi je suis pour que, chaque agent ayant les mêmes habiletés... tu vois les mêmes capacités physiques euh... ait un traitement identique... à partir du moment où ils sont aptes à faire une tâche, y'a pas de raison que leur traitement soit différent. Et c'est dans cette logique-là que... Alors ça c'est mon premier point, le deuxième point c'est : cet agent connaît bien la benne parce qu'il l'a souvent faite, donc ça veut dire que finalement si on reste sur le fait que ce soit cet agent qui fasse le maximum cette benne, on se prive de cette possibilité que d'autres agents en la faisant souvent puisse retenir eux-mêmes cette benne.

25. Chercheur : D'accord... alors qu'est-ce que tu en penses de ça ?

26. Bakary : Nan je suis pas d'accord parce que les autres c'est pas qu'ils la font pas du tout... ils la font... mais, comment dirais-je, à une fréquence beaucoup moindre par rapport à lui...

27. Alexandre : Du coup ils peuvent pas la retenir alors...

28. Bakary : ben si ! Comme je te disais la fois, on a euh... monsieur F. qui est là en moins de deux ans, il connaît tous les, tous les itinéraires.

Tableau 1 : Extrait d'autoconfrontation croisée entre Alexandre et Bakary

Table 1: Extract from a crossed self-confrontation between Alexandre and Bakary

Alors que les deux chefs d'équipes cherchent à résoudre dans le dialogue le problème de la prise en compte de l'engagement subjectif des éboueurs lors de la répartition des tâches, une controverse s'engage entre eux. Pour Bakary, le principe d'équité constitue le critère 
principal de la répartition: il faut attribuer les tâches en fonction des performances des agents, gage d'un travail de qualité. Pour Alexandre, à l'inverse, l'égalité est le premier critère à prendre en compte : affecter les tâches indistinctement à tous les éboueurs, quelle que soit leur expérience. Ce critère permettrait, selon lui, de ne pas démotiver les jeunes éboueurs, sans expérience, qui pourraient avoir le sentiment d'un management par «traitement de faveur » avec les plus expérimentés.

Deux mouvements discursifs principaux peuvent être identifiés dans cette séquence. L'analyse de l'extrait sera organisée en fonction de chacun de ces deux mouvements.

Le chercheur tente d'abord, en s'adressant à Bakary (en 1), d'introduire la question de l'efficacité dans le débat qui s'instaure. Bakary s'en empare afin de poursuivre l'argumentation («notre grand souci c'est que les collectes soient finies», en 2). Bakary peut soutenir ici que sa manière d'affecter D., qu'il considère comme un éboueur très performant, sur ses journées de $9 \mathrm{~h}$ à la VE permet de terminer la collecte de façon systématique. Mais pour Alexandre, le pendant de ce système d'affectation (affecter un autre éboueur que D. sur la VE) n'a pas été suffisamment éprouvé pour être en mesure d'en tirer des conclusions du côté de l'efficacité («[L'échec de la collecte] n'est arrivé qu'une fois », en 3 et 5). Lors des journées de $6 \mathrm{~h}$, et lorsque D. est en repos, ils ont l'occasion d'expérimenter des binômes d'éboueurs sur la VE en l'absence de D. Pour cette raison, Bakary indique que l'efficacité de la pratique doit être évaluée sur un temps plus long (« ça ne fait pas longtemps qu'on a opté ce changement»).

À ce point de la conversation, et alors que l'efficacité de la collecte est envisagée uniquement sous l'angle de l'expérience et de la performance de D., Alexandre introduit la question du collectif d'éboueur ( « sur la totalité », en 7). Autrement dit, en affectant D. sur la VE de façon systématique sur les journées de 9 h, Alexandre fait l'hypothèse d'un effet sur l'équipe d'éboueur qui se sent mise à l'écart de la tâche de VE, alors que D. y est valorisé, ce qui représente un obstacle indirect à l'efficacité de la collecte et à l'engagement des éboueurs. Afin de poursuivre l'argumentation sur ce nouvel objet Bakary rapatrie dans la discussion une règle générique de gestion de l'équipe dans l'activité d'encadrement : l'équité («on devrait tous les traiter de façon équitable », en 8). Cette généricité se signale dans le dialogue par l'usage du « on », en référence au collectif de métier. En effet, dans le milieu, il est admis que l'activité de répartition des tâches doive tenir compte des préférences des uns et des autres, afin que chaque éboueur puisse se sentir satisfait des tâches qui lui sont attribuées, et ce afin de maintenir chez chacun un niveau de motivation suffisant à la bonne réalisation du travail. Mais, dès le tour de parole suivant (en 9), Alexandre propose une alternative au traitement équitable : " ou égale ouais ».

Cette alternative n'est pas tout de suite perçue par Bakary qui continue à suivre le fil de sa pensée («voilà égale », en 10) en tentant d'unifier dans une même signification ces deux alternatives possibles. Alexandre, qui tient à faire la distinction, reprend Bakary et insiste sur le fait qu'il préfère qualifier le traitement des éboueurs comme «égal » plutôt que comme « équitable» (en 11). Le chercheur s'engage alors sur la voie déjà foulée par Bakary, en agglomérant à son tour les significations des deux termes («Et donc toi ton souci c'est que ce soit équitable quoi ? », en 14). Mais Alexandre continue de résister à cette assimilation, cette fois en direction du chercheur qui ratifiera la distinction (en 16) entre le critère d'égalité et le critère d'équité. De notre point de vue, c'est au cours de ces derniers tours de paroles (10 à 16) que s'opère la segmentation de l'activité des deux professionnels-chefs d'équipe, dans la définition par le dialogue de la distinction entre le critère d'équité et le critère d'égalité, distinction qui n'avait jamais été posée auparavant dans l'intervention.

Ce premier mouvement (de 1 à 16) correspond à la construction d'un espace de dialogue autour de la question managériale de prise en compte de l'engagement des éboueurs lors de la répartition des tâches au sein de l'équipe. Les tours de paroles successifs permettent aux deux professionnels et au chercheur de poser ensemble dans l'interlocution l'objet qui sera conjointement pris en charge ensuite. En l'occurrence, c'est l'opposition entre deux critères, « équité » ou «égalité » dans la répartition des tâches, qui constituera le thème central dans 
la poursuite du dialogue. L'interlocution prend également la forme d'une dispute professionnelle ou controverse, au cours de laquelle le conflit de critères est mis au travail. La controverse constitue ici une forme de réalisation langagière du conflit de critères équité/égalité dans l'activité de répartition des tâches des deux professionnels.

Le second mouvement que nous identifions dans cet extrait de dialogue (de 17 à 28) permet à Bakary et Alexandre de mieux circonscrire la distinction précédemment opérée entre l'équité et l'égalité. Afin d'approfondir cette distinction, le chercheur tente de produire une interprétation de ce qu'Alexandre peut entendre par «égal». Mais Bakary, qui semble affecté par l'alternative proposée, coupe le chercheur afin de pousser Alexandre lui-même à lui attribuer une signification (« Pourquoi pas équitable ?», en 19).

Dans la suite de la conversation Alexandre développe ce qu'il entend par «égal » en y associant un certain nombre de critères tels que les « habiletés », les « capacités physiques », les « aptitudes » des éboueurs, mais aussi « connaître [l'itinéraire de] la benne » ou « donner la possibilité aux autres de connaître cet itinéraire».

La controverse ainsi entamée se poursuit jusqu'à la fin de la séquence en autoconfrontation croisée, sur près de 30 minutes, au cours desquelles Bakary et Alexandre redéfinissent ce qu'ils cherchent à faire lorsqu'ils attribuent les tâches aux uns ou aux autres des éboueurs.

\subsection{3.- La reprise collective de la segmentation}

Suite à cette première séquence et avec l'accord de Bakary et Alexandre, le chercheur a proposé de reprendre collectivement l'extrait de dialogue précédemment présenté. Il a ainsi été visionné par l'ensemble des chefs d'équipe engagés dans le dispositif d'intervention. Suite à la présentation de l'extrait, une conversation collective s'engage entre les professionnels. Nous ne ferons que décrire ici la teneur de ce dialogue collectif, dans un souci de synthèse.

Au cours de cette reprise collective, les chefs d'équipe sont revenus sur les critères de l'égalité et de l'équité d'abord mis en lumière dans le dialogue entre Bakary et Alexandre. Chacun a pu dire aux autres laquelle de ces deux modalités il préférait réaliser dans sa propre activité. Se faisant, un dialogue s'est réalisé entre eux, par exemple à propos des possibilités réelles de mettre en œuvre ces critères : au nom de l'égalité dans la répartition des tâches, peut-on réellement affecter un éboueur sur une tâche qu'il n'aime pas faire, qu'il ne sait parfois pas réaliser, et qu'il n'a pas toujours envie de faire? Au nom de l'équité, peut-on réellement fonctionner uniquement en attribuant les tâches les plus valorisantes aux éboueurs les plus performants?

Dans cette activité dialogique collective, les controverses et les questions de métier ont permis à chacun de s'approprier la segmentation de leur activité réalisée dans le dialogue entre Alexandre et Bakary.

\subsection{4.- La segmentation comme voie d'interprétation de l'activité}

L'observation du travail, lorsqu'elle est réalisée par les professionnels eux-mêmes, les conduit à faire émerger dans le dialogue de nouveaux critères de réalisation du travail. L'observation fait apparaître de nouvelles alternatives possibles, potentiellement disponibles pour les réalisations à venir. C'est ce que nous appelons la segmentation de l'activité. Nous cherchons à qualifier le devenir de cette segmentation dans les dialogues tenus ultérieurement par les professionnels.

Nous nous appuierons ici sur un extrait de dialogue entre deux chefs d'équipe ayant vécu l'émergence de la segmentation équité/égalité, qui fait maintenant objet de débat au sein du collectif professionnel, pour tenter d'en dire quelque chose du point de vue du développement de leur activité d'analyse. Les extraits d'autoconfrontation simple et croisée que nous présentons ci-dessous se déroulent donc a posteriori des dialogues précédemment décrits. Ils doivent nous permettre de montrer que la segmentation laisse des traces dans l'activité dialogique à venir des professionnels, et qu'elle est ainsi mise à l'épreuve au cours 
d'observations ultérieures de leur activité. Elle constitue, en somme, une nouvelle ressource pour eux afin de repenser leur activité.

Les dialogues ultérieurement réalisés s'organisent en deux parties distinctes. Le premier dialogue que nous présentons (extrait $\mathrm{n}^{\circ} 2$ ) est issu d'une autoconfrontation simple avec Nadine, une professionnelle-chef d'équipe depuis dix ans. Le second dialogue (extrait $n^{\circ} 3$ ) est un extrait d'une autoconfrontation croisée entre Nadine et Julien, chef d'équipe depuis cinq ans.

Le film d'activité travaillé en autoconfrontation simple présente l'activité de Nadine, au moment où elle doit mettre en route le travail des éboueurs. C'est aussi à ce moment-là que Nadine revient sur le tableau de répartition des tâches, pour y apporter les modifications de dernière minute. Dans le dialogue avec le chercheur en autoconfrontation simple, Nadine revient sur les critères qui guident la répartition des tâches dans son activité.

1. Nadine : Bien souvent euh Lucien il me dit euh vendredi tu peux pas me mettre un peu aux $\mathrm{GO}^{4}$ euh ? C'est pour ça il vient me voir moi.

2. Chercheur : ah ouais d'accord

3. Nadine : mais bon je sais que je peux compter sur lui donc... donc de temps en temps je le mets aux GO...

4. Chercheur : parce que toi quand tu fais ça, quand tu... quand tu mets en pied par exemple ça part du principe que... c'est... ça les préserve ?

5. Nadine : oui ça les préserve, ça... euh... pour eux c'est une responsabilité c'est... c'est un peu les... les promouvoir quelque part hein. C'est, ils sont responsables, les gens les connaissent bien souvent quand ils sont en pied quelque part... les restos les connaissent...

6. Chercheur : et ça ça se mérite c'est ça ?

7. Nadine : oui un peu ouais...

8. Chercheur : ouais, d'accord... toi tu serais plutôt d'accord, tu te rappelles la vidéo avec Bakary et Alexandre ? sur le... où lui il mettait le même agent sur une benne sur ses deux 9 heures euh... parce qu'il faisait bien le boulot, il «pouvait compter sur lui » c'est ça qu'il dit.

9. Nadine : oui... et puis y'avait l'histoire... ça m'avait... ça m'avait fait... ça m'avait interpellé quand même quelque part... tu sais comment c'était y'en a un qui disait attend... les deux... les deux euh... comment il disait y'en a un qui disait, tu sais les deux, les deux mots... je cherche les deux mots...

10. Chercheur : euh... équité égalité ?

11. Nadine : équité égalité. Hmm, moi je dirai équité, je dirai pas égalité...

12. Chercheur : équité... et dans quel sens euh...

13. Nadine : tu peux pas... tu peux pas attribuer le même travail à deux gars... enfin le même travail... c'est... la... c'est pas ça. Il faut être juste. Faut pas être euh... si tu veux et l'équité dans le mot équité moi je dis qu'il y a une idée de justice. Tu es juste, le gars qui est bon tu vas être plus juste avec lui qu'avec un gars qui est pas bon puisque il est pas juste avec toi. Tu vois ce que je veux te dire?

14. Chercheur : hmm, oui c'est intéressant ça.

15. Nadine : et justement moi j'étais plus pour l'équité que pour l'égalité... et j'avais bien aimé cette discussion qu'ils avaient eue tous les deux là...

Tableau 2 : Extrait d'autoconfrontation simple avec Nadine

Table 2: Extract from individual auto-confrontation with Nadine

4 GO = Gros Objets. L'abréviation désigne la tâche de collecte des « objets encombrants ». 
Dans cette séquence, Nadine explique au chercheur ce qui la pousse à attribuer des secteurs «en pied ${ }^{5}$ à certains éboueurs. En 4 , le chercheur rapatrie une hypothèse déjà mise en discussion avec d'autres chefs d'équipe au cours de l'intervention («quand tu mets en pied par exemple ça part du principe que... c'est... ça les préserve ? »). En 5, Nadine envisage la préservation du côté de la responsabilité qu'on confie à l'éboueur, et de l'effet de satisfaction supposé que cela peut produire chez lui («C'est, ils sont responsables, les gens les connaissent bien souvent quand ils sont en pied quelque part »). En 8, le chercheur tente de pousser Nadine à reprendre la question de l'affectation «en pied» à partir du dialogue réalisé entre Bakary et Alexandre. En effet, l'affectation «au mérite », évoquée en 6 par le chercheur, et validée en 7 par Nadine, l'amène à comparer les deux situations (en 8) qui semblent identiques selon le même critère d'équité. En 9, Nadine tente de retrouver les termes de la segmentation, sans y parvenir. Afin de soutenir Nadine dans cette élaboration, le chercheur reprécise les deux termes évoqués lors de la discussion collective précédente en 10. Cette actualisation de la segmentation pousse Nadine, en 11 et en 15, à prendre position du côté de l'équité. Elle indiquera en 13 les critères qui sous-tendent, selon elle, une manière de répartir les tâches équitablement.

Cet extrait de dialogue entre le chercheur et Nadine a une fonction d'actualisation de la segmentation. Le dialogue permet ici à Nadine de réaffirmer son positionnement du côté de l'équité, tout en engageant pour elle-même une élaboration autour des critères qui pourraient guider une répartition des tâches équitable.

Dans l'extrait suivant, le même film est présenté aux deux professionnels, Nadine et Julien. Le film d'activité de Julien est également visionné. Julien réalise son activité dans la même situation que Nadine, lors de la mise en route du travail dans son atelier.

1. Nadine : par exemple s' il rentre de repos ils savent pas... en principe quand il rentre de repos il laisse gérer plus le chef qui était là la veille... tu vois.

2. Julien : hmm hmm

3. Nadine : par exemple moi je suis là... je suis de repos... je serai pas là lundi, mais demain je préparerai pas le travail.

4. Julien : à moins que tu sois toute seule

5. Nadine : sauf si mon collègue est pas là, à moins que je sois toute seule. Tu vois. Mais en principe je prépare pas le travail si je suis pas là le lendemain, parce que mon collègue il a sa façon de faire je le laisse faire et puis, dans la mesure où mon collègue qui était là la veille n'est pas là le jour où j'arrive euh... ben je respecte le travail qu'il m'a mis, je vais pas changer son travail si tu veux...

6. Chercheur : hmm et du coup, quand ça arrive ça par exemple, est-ce que tu constates que... bah je sais pas, monsieur X, toi tu le mets toujours sur cette tâche-là enfin, je caricature hein... et puis euh... mon collègue lui et ben il l'a mis sur autre chose. Ça arrive ça ?

7. Nadine : oui ça m'arrive de constater.

8. Chercheur : d'accord... et ça c'est euh... c'est quoi ça c'est bien c'est pas bien ?

9. Nadine : ça dépend qui. Bon ça dépend du collègue... bon comment il fait mon collègue il a tendance à... à être je dirai euh... à faire l'égalité et moi l'équité. Donc on est pas forcément d'accord sur euh sur les points... tu vois ce que je veux te dire?

10. Chercheur : oui c'est-à-dire que lui euh... il tient moins compte des différences entre les agents ?

11. Nadine : voilà... moi je fais peut-être plus de différences oui.

5 L'expression « en pied » signifie l'attribution systématique d'un secteur de travail à un même éboueur. Le plus souvent les secteurs sont attribués indistinctement entre les éboueurs, afin de faire varier leur activité. 
12. Chercheur : par exemple il pourrait mettre un que tu dis « léger» là... il pourrait le mettre sur un canton où toi tu l'aurais pas mis.

13. Nadine : moi je l'ai vu mettre sur les $\mathrm{GO}^{6}$ alors que je supporte pas ça... le gars il a 90 jours de présence, le jour où il vient, moi je le mettrai jamais aux GO, jamais. Et puis lui il le fait, tu vois...

14. Julien : il peut en profiter pour faire des $\mathrm{AT}^{7}$ machin...

15. Nadine : ouais, et il m'a fait un AT.

16. Julien : ah ben tu vois.

17. Nadine : j'suis contente parce qu'il m'a fait l'AT (rires)

18. Chercheur : ça a servi de leçon c'est ça que tu veux dire ?

19. Nadine : j'espère que mon collègue va comprendre, mais... j'ai pas l'impression...

20. Julien : oui c'est risqué ça

21. Chercheur : toi aussi ça arrive ça ?

22. Julien : oui... hmm

Tableau 3 : Extrait d'autoconfrontation croisée entre Nadine et Julien

Table 3: Extract from a crossed self-confrontation between Nadine and Julien

Le début de l'extrait (tours de paroles 1 à 5) fait état de l'objet de discours en cours d'élaboration entre les deux professionnels. Cet objet concerne la coordination entre deux chefs d'équipe d'un même atelier dans la préparation du tableau de répartition des tâches. Avant de partir pour ses jours de repos, Nadine ne prépare pas le travail, elle laisse son collègue le faire, afin qu'il puisse lui-même gérer les éventuelles modifications à y apporter le lendemain. De plus, les chefs d'équipe ayant chacun leur façon de répartir les tâches, Nadine estime devoir respecter les manières de faire de son collègue en le laissant réaliser lui-même la répartition des tâches (en 5). Afin de mettre au travail entre eux cette question des manières différentes de préparer le tableau de répartition entre collègues, le chercheur propose en 6 d'envisager la situation, qui peut parfois arriver (en 7), dans laquelle le collègue prépare la répartition pour eux, et que cette répartition leur pose problème. À cette occasion, Nadine réalise en 9 une reprise de la distinction entre égalité et équité afin de soutenir son point de vue auprès de Julien et du chercheur. Elle fait ici usage de la distinction, qui fonctionne désormais comme un concept de métier, pour caractériser les différences qu'elle constate entre ses manières de faire le tableau de répartition et celles de son collègue («mon collègue il a tendance à... à être je dirai euh... à faire l'égalité et moi l'équité »). Ce faisant, ce qui pourrait être attribué à un trait de caractère, ou à une disposition à bien savoir répartir les tâches, est ici attribué par Nadine à un choix délibéré et à un désaccord sur les critères de qualité. En effet, en énonçant la modalité de répartition mise en œuvre par son collègue, elle réalise dans le même temps une différenciation entre leurs manières de faire, qu'elle peut alors attribuer aux différentes variantes précédemment élaborées collectivement et individuellement (extrait 1). Autrement dit, c'est parce qu'elle a pu élaborer son activité à partir de la segmentation opérée dans les dialogues précédents, qu'elle peut maintenant, dans le cours de la conversation, en disposer pour produire une interprétation de ce qu'elle est en train d'observer. Pour cette raison, on peut dire que Nadine s'est saisie de la conceptualisation équité/égalité, afin de segmenter un nouvel objet dans son activité : les manières différentes de faire le travail entre collègues.

\footnotetext{
6 《gros objets »= tâche de ramassage des objets encombrants

7 Accident du Travail
} 


\section{3.- Conclusion}

Au terme de l'exposé des matériaux cliniques, nous pouvons à présent revenir sur les premiers éléments qui ont guidé l'élaboration théorique.

C'est dans le premier extrait présenté que s'opère une segmentation de l'activité, faisant ainsi émerger deux critères possibles qui tiennent compte de manière différente de l'engagement subjectif des éboueurs dans leur travail. Dans l'extrait $n^{\circ} 1$, les observations de Bakary sur l'une des règles générique du métier de chef d'équipe - l'équité dans la répartition des tâches - provoquent chez Alexandre un effet de distanciation. Il ne se reconnait plus tout à fait dans l'activité telle que son collègue la décrit, et réagit à son interprétation. À ce stade, les observations réalisées par Bakary, qui appartiennent à son espace subjectif, pour le dire à la manière de Droz, sont des certitudes ou, du moins, elles peuvent l'être. Mais puisqu'elles sont insérées dans un espace intersubjectif dans lequel Alexandre est impliqué, ces observations sont rendues discutables dès lors que l'un ou l'autre ne reconnaît plus son activité propre dans les mots d'autrui. C'est pourquoi Alexandre propose une autre voie possible pour envisager la répartition des tâches, l'égalité. Dès lors, la tâche ne consiste plus seulement à commenter l'activité du collègue, mais aussi à tenter de résoudre les conflits de l'activité de répartition des tâches, en tant qu'elle peut constituer un moyen pour favoriser l'engagement subjectif des éboueurs dans la réalisation de leur travail. On pourrait dire que l'activité discursive change de destinataire au cours de l'observation, qu'elle change d'interlocuteur. Elle ne s'adresse plus seulement à l'activité de l'un ou à l'activité de l'autre, mais aussi, et surtout à ce qu'il y a de générique dans le métier, comme instrument pour faire ce qui est à faire, c'est-à-dire au genre professionnel en vigueur (Clot, 1999 ; 2008). C'est ce nouvel interlocuteur qui, dans le dialogue entre Alexandre et Bakary, « exige pour se laisser convaincre ». En instruisant la possibilité d'une alternative possible à l'équité, c'est le genre professionnel qui s'en trouve potentiellement transformé, car il s'opère alors un accroissement des possibles dans l'activité de répartition des tâches pour chaque chef d'équipe.

La distinction opérée entre "égalité » et «équité » au cours de la conversation constitue une segmentation potentielle de l'activité de chacun des chefs d'équipe, qui peut être rendue disponible à tous dans la poursuite du travail d'analyse par l'ensemble du groupe de pairs engagé dans le dispositif. L'effet à priori de cette segmentation ne peut pas être spécifié, c'est pourquoi nous insistons sur la potentialité de la segmentation. Pour devenir effective, la distinction opérée doit devenir un instrument pour l'activité propre des autres chefs d'équipe, ce qui ne va pas de soi.

Les deux derniers extraits présentés (extraits $n^{\circ} 2$ et 3 ) montrent comment la segmentation concourt au développement de l'observation. Pour Nadine, elle constitue un moyen de poursuivre l'analyse des situations de travail dans le cours des autoconfrontations. Mais pas uniquement. Elle peut également être opérante pour repenser d'autres activités que celle de répartition des tâches, comme c'est le cas ici pour Nadine. Dans ce cas, elle fonctionne comme un concept de métier, "prêt à l'emploi », et permet aux professionnels de produire des interprétations nouvelles à partir des observations réalisées précédemment.

Dans le processus de segmentation que nous avons décrit au travers des trois extraits de dialogues présentés, l'observation remplit une diversité de fonctions. Elle est d'abord un moyen pour le chercheur de mettre au travail chez le sujet observé de nouveaux objets en tentant de provoquer chez lui un dialogue intérieur. Elle est ensuite un moyen pour le sujet lui-même, en autoconfrontation simple, lorsqu'il tente d'expliquer au chercheur, et à luimême, la manière dont il s'y prend pour agir en situation. Enfin, elle constitue un moyen d'élaboration de l'interprétation des sujets lorsqu'elle leur permet d'adresser leurs analyses à un autre professionnel en autoconfrontation croisée.

De plus, l'observation, lorsqu'elle constitue l'instrument des professionnels entre eux, et pas uniquement celui du chercheur, appelle de nouvelles observations. Dans l'exemple que nous avons présenté, l'activité d'observation réalisée par les professionnels procède par sélection 
de segments de leur activité qui contiennent une multitude d'objets de dialogue potentiels, c'est le processus de segmentation que nous avons identifié. En observant le tableau de répartition des tâches réalisé par Alexandre dans un cadre qui favorise l'analyse, les deux professionnels peuvent poser la question de l'équité comme un objet en soi dans le cours de l'autoconfrontation. De la même manière, en observant sa propre activité sur les images filmées, Nadine peut regarder la question de l'hétérogénéité du collectif à partir de la question de l'équité, en segmentant à nouveau son activité du côté des manières de faire différentes entre collègues.

Ces remarques nous amènent à concevoir l'observation comme une activité en développement dans le déroulement de la méthode en autoconfrontation croisée. D'abord source de développement du dialogue intérieur, elle devient ressource pour le développement $\mathrm{du}$ dialogue dans le collectif. Par les segmentations successives qu'elle occasionne, l'observation se fait de plus en plus minutieuse, elle devient un moyen d'entrer dans le détail des analyses à la recherche de l'objectivité, qui, s'il elle est déterminée pour un temps, pourra être reprise à l'occasion d'observations ultérieures. De ce point de vue, la répétition des observations d'un contexte à un autre constitue une ressource pour faire "varier la focale » des observations à venir. Elles permettent aux professionnels d'observer à nouveau la même situation en faisant des observations précédentes le moyen d'y retrouver autre chose que ce qu'on a déjà pu en dire.

L'observation, d'abord source de production des interprétations réalisées par les professionnels, devient, suite aux segmentations de l'activité pratique qui s'opèrent dans le dialogue, ressource pour produire de nouvelles interprétations. Il s'établit ainsi un cycle de l'observation au cours de l'intervention, de source à ressource, et inversement. Mais pour que ce cycle puisse s'établir, le rapport initial observateur/observé doit migrer dans le déroulement de la méthode, d'abord entre le chercheur et le professionnel, il devient ensuite un moyen pour les professionnels entre eux, de questionner le « déjà dit », c'est-à-dire de réinterroger ce qui fait «fond commun » dans le métier. Dans le cours des situations où ils sont amenés à s'observer, le rapport intersubjectif constitue donc un moyen privilégié pour donner une histoire à l'objectivité. La répétition des situations intersubjectives d'observation permet de construire de l'objectivité en redéfinissant entre les professionnels un périmètre dialogique qu'ils ne soupçonnaient pas, c'est-à-dire en réévaluant les critères de qualité considérés comme acquis. C'est le cas ici lorsqu'Alexandre et Bakary redécouvrent une alternative possible à une répartition des tâches équitable, puis lorsque cette alternative est rediscutée avec l'ensemble du collectif. Car l'équité fait partie dans ce milieu du discours entretenu par chaque chef d'équipe lorsqu'on l'interroge sur sa manière de réaliser le travail - «faire une répartition équitable». C'est pourquoi la distinction opérée par les chefs d'équipe entre équité et égalité revêt de notre point de vue une fonction psychologique centrale lorsqu'on tente de provoquer, en clinique de l'activité, le développement de l'activité des professionnels concernés. Cette fonction peut être exprimée de la manière suivante : en opérant une segmentation à partir d'une équité «ancrée » dans le discours des professionnels, vers deux modalités d'action nouvelles, l'équité ou l'égalité, c'est le genre professionnel qui s'en trouve potentiellement modifié. Là où il n'y avait auparavant qu'une seule manière de faire ou de dire, chacun peut à présent se "positionner » autour de deux variantes distinctes. C'est donc le «stock de "mise en actes", de "mise en mot" mais aussi de conceptualisations pragmatiques (Samurçay \& Pastré, 1995), "prêts à servir" » (Clot, 2008, p. 107) qui s'en trouve renouvelé. On pourrait dire qu'il s'agit là d'une forme de renouvellement du «pré-travaillé social», une modification des conditions initiales de l'activité en cours, préalables indispensables de l'action.

Enfin, la répétition des observations à travers des contextes multiples fonctionne comme un instrument psychologique de délimitation du réel. La segmentation du drame et la formation des faits psychologiques (Politzer, 1928/1967), produits de l'activité d'observation, semblent alors fonctionner comme «catalyseurs ", permettant de prendre position face à un objet mieux circonscrit via l'activité d'analyse, de faire des choix de positionnement, et donc de 
construire la responsabilité de ses actes en assumant de trancher dans l'étendue des possibles.

Pour terminer, nous voudrions souligner que, dans cette perspective en clinique de l'activité, l'usage clinique de l'observation n'est pas tourné entièrement vers le recueil de données. L'observation est d'abord tournée, dans l'intervention, vers la production d'une genèse instrumentale (Rabardel, 1995) particulière, qui fait de l'observation un instrument à disposition des professionnels pour revitaliser le dialogue à l'intérieur du collectif en développant les objets et les destinataires de leur activité, et au-delà, dans l'organisation. C'est pourquoi l'observation est utilisée ici dans différents registres successifs. Pour que l'activité d'observation puisse se déplacer du chercheur aux professionnels entre eux, cette traversée des registres est indispensable. Elle permet de faire varier les destinataires de cette activité à l'intérieur de chacune des phases de la méthode. Ici « la connaissance n'est plus la découverte des nécessités ou des évidences, mais l'actualisation des possibles ou la création de nouveaux possibles » (Ciccone, 2013, p. 21).

La manière d'engager les professionnels dans une segmentation du drame et dans l'émergence de faits psychologiques en développant l'observation entre eux nous paraît constituer un moyen de pousser le collectif à se dépasser lui-même, c'est-à-dire à repousser l'objectivité, en prenant des libertés avec le «déjà-dit»et le «déjà fait»dans le milieu professionnel.

\section{BIBLIOGRAPHIE}

Arborio, A.-M. (2007). L'observation directe en sociologie : quelques réflexions méthodologiques à propos de travaux de recherches sur le terrain hospitalier. Recherche en soins infirmiers, 3(90), 2634.

Bakhtine, M. (1984). Esthétique de la création verbale. Paris: Gallimard.

Bunge, M. (1967/1984). L'observation. In M.P. Michiels-Philippe (Ed.), L'observation (pp. 47-60). Neuchâtel: Delachaux \& Niestlé.

Chapoulie, J.-M. (2000). Le travail de terrain, l'observation des actions et des interactions, et la sociologie. Sociétés contemporaines, 40, 5-27.

Ciccone, A. (2013). L'observation clinique. Paris: Dunod.

Clot, Y. (1999). La fonction psychologique du travail. Paris: PUF.

Clot, Y. (2003). Vygotski, la conscience comme liaison. In L. Vygotski : Conscience, inconscient, émotions (pp. 7-59). Paris : La dispute.

Clot, Y. (2005). L'auto-confrontation croisée en analyse du travail : l'apport de la théorie bakhtinienne du dialogue. In, L. Filliettaz \& J. P. Bronckart (Eds.), L'analyse des actions et des discours en situation de travail (pp. 37-55). Louvain-La-Neuve: Peeters.

Clot, Y. (2008). Travail et pouvoir d'agir. Paris: PUF.

Clot, Y., \& Faïta, D. (2000). Genres et styles en analyses du travail : concepts et méthodes. Travailler, 4,7-43.

Clot, Y., Faïta D., Fernandez, G., \& Scheller, L. (2001). Les entretiens en auto-confrontation croisée : une méthode en clinique de l'activité. Éducation permanente, 146, 17-27.

Clot, Y., \& Fernandez, G. (2005). Analyse psychologique du mouvement : apport à la compréhension des TMS. Activités, 2(2), 69-78.

Darwin, C. (1984). L'expression du mépris ou du défi. In M.P. Michiels-Philippe (Ed.), L'observation (pp. 137-140). Neuchâtel: Delachaux \& Niestlé.

Droz, R. (1984). Observations sur l'observation. In M.P. Michiels-Philippe (Ed.), L'observation (pp. 7-30). Neuchâtel: Delachaux \& Niestlé.

Duboscq, J., \& Clot, Y. (2010). L'autoconfrontation croisée comme instrument d'action au travers du dialogue : objets, adresses et gestes renouvelés. Revue d'anthropologie des connaissances, 4(2), 255-286.

Kostulski, K. (2011). Development of Activity through Reflection: The case of the public prosecutor's lapsus linguae. In D. Nicolini \& O. Eikeland (Eds). Changing practices through reflection, Journal 
of Organisation and Change Management, 24(2), 191-211.

Kostulski, K. \& Clot, Y. (2007). Interaction et migration fonctionnelle : Un développement en autoconfrontation croisée. In Y. Clot \& K. Kostulski (Eds.), Dialogue, activité, développement. Psychologie de l'Interaction (pp. 73-108). Paris: L'Harmattan.

Lapassade, G. (2002). L'observation participante. In J. Barus-Michel, E. Enriquez, \& A. Lévy (Eds.), Vocabulaire de psychosociologie (pp. 375-390). Erès.

Mollo, V., \& Falzon, P. (2004). Auto- and allo-confrontation as tools for reflective activities. Applied Ergonomics, 35(6), 531-540.

Norimatsu, H., (2014). Initiation aux techniques d'observation. In H. Norimatsu \& N., Pigem (Eds.). Les techniques d'observation en sciences humaines (pp. 5-45). Paris: Armand Colin.

Pacaud, S. (1954). Analyse psychologique et psychophysiologique du travail. In H. Piéron, M. Reuchlin, R. Bize, C. Benassy-Chauffard, S. Pacaud, \& P. Rennes (Eds.), Traité de psychologie appliquée. Livre 3 : L'utilisation des aptitudes. Orientation et sélection professionnelle (pp. 579-619). Paris: PUF.

Perrot, E., Bonnemain, A., \& Kostulski, K. (2013). L'observation du travail en clinique de l'activité : ressource dialogique au service du développement de l'activité des professionnels. In Actes du colloque international DIM-GESTES, "Quelles actions pour un autre travail : soulager la souffrance, éradiquer les facteurs de risques, "soigner le travail” ? », 10-11 juin 2013.

Politzer, G. (1928/1967). Critique des fondements de la psychologie. Paris: PUF.

Rabardel, P. (1995). Les hommes et les technologies. Approche cognitive des instruments contemporains. Paris: Armand Colin.

Rimé, B. (2005). Le partage social des émotions. Paris: PUF.

Samurçay, R., \& Pastré, P. (1995). La conceptualisation des situations de travail dans la formation des compétences au travail. Éducation permanente, 123, 34-42.

Simonet, P. (2011). L'hypo-socialisation du mouvement. Prévention durable des troubles musculosquelettiques chez les fossoyeurs municipaux. Thèse de doctorat en psychologie du travail. Paris: CNAM.

Simonet, P., Caroly, S., \& Clot, Y. (2011). Méthodes d'observation de l'activité de travail et prévention durable des TMS : action et discussion interdisciplinaire entre clinique de l'activité et ergonomie. Activités, 8(1), 104-128.

Volochinov, V.N. (1977). Le marxisme et la philosophie du langage. Paris: Éditions de minuit.

Vygotski, L. (1978). Mind in society: the development of higher psychological processes. Cambridge: Harvard University Press.

Vygotski, L. (1997). Pensée et langage. Paris: La dispute.

Vygotski, L. (1999). La signification historique de la crise en psychologie. (Barras \& Barberies, Trad.). Lausanne et Paris: Delachaux et Niestlé.

Vygotski, L. (2003). Conscience, inconscient, émotions. (F. Sève \& G. Fernandez, Trad.). Paris: La Dispute.

Wallon, H. (1983). Les origines du caractère chez l'enfant. Paris: PUF.

\section{RÉSUMÉ}

L'objet de cet article est d'analyser le développement de l'activité d'observation dans la méthode en autoconfrontation croisée. La première partie reprend les éléments théoriques relatifs à l'observation et à l'intersubjectivité qui s'y déploie. La seconde partie présente un exemple d'intervention réalisée avec la méthode des autoconfrontation croisée en clinique de l'activité. Nous étudions à partir de cet exemple les effets du développement de l'observation lorsqu'elle se réalise entre les professionnels, et non pas entre le chercheur et le sujet observé. Nous présentons comment la migration de l'intersubjectivité dans le processus 
d'observation affecte la manière dont les professionnels peuvent penser leur travail. Plus précisément, nous proposons de concevoir l'observation comme un moyen pour les professionnels de «segmenter » leur activité, et de construire ainsi, individuellement et collectivement, de nouveaux objets de pensée dans le cours de l'intervention.

\section{MOTS-CLÉS}

méthodes d'observation, intersubjectivité, autoconfrontation, intervention, clinique de l'activité.

\section{RÉFÉRENCEMENT}

Bonnemain, A., Perrot, E., \& Kostulski, K. (2015). Le processus d'observation, son développement et ses effets dans la méthode des autoconfrontations croisées en clinique de l'activité. Activités, 12(2), 98-124. http://www.activites.org/v12n2/v12n2.pdf

Article soumis le 15/07/2014, accepté le 21/06/2015 